\title{
La mitose \\ sous surveillance
}

\author{
Anna Castro, Suzanne Vigneron, \\ Thierry Lorca, Jean-Claude Labbé
}

$>$ Le point de contrôle mitotique est essentiel pour permettre une répartition équitable du matériel génétique au cours de la mitose. Il prévient le déclenchement de l'anaphase jusqu'à ce que tous les chromosomes soient attachés au fuseau mitotique et alignés sur la plaque équatoriale. Sa fonction est d'empêcher une ubiquitine ligase - l'anaphase promoting complex (APC) - d'ubiquitinyler certaines protéines dont la dégradation est nécessaire à la mise en route de l'anaphase. Chez les eucaryotes supérieurs, la perte de ce point de contrôle peut conduire à une mauvaise répartition des chromosomes, et contribuer ainsi à l'instabilité génomique observée dans la plupart des cellules tumorales. <

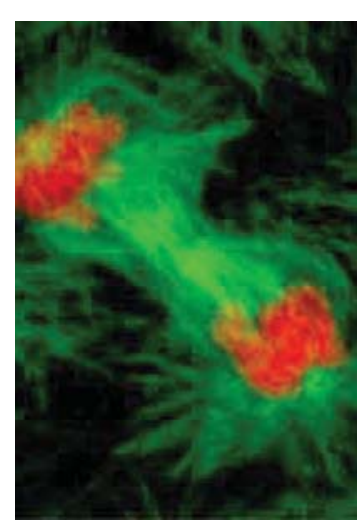

tubules et de l'activité de moteurs moléculaires, les kinésines [1]. Pendant cette période, les chromatides sœurs de chaque chromosome sont capturées par des microtubules émanant des pôles opposés du fuseau. Cette capture se produit par l'intermédiaire d'une structure protéique assemblée sur l'ADN centromérique des chromatides, le kinétochore. Une fois l'attachement bipolaire acquis, les chromosomes s'alignent sur la plaque équatoriale du fuseau: la métaphase correspond au moment où tous les chromosomes sont alignés. Pendant l'anaphase, les chromatides sœurs migrent vers les pôles opposés du fuseau.

La transition métaphase/anaphase (M/A) n'intervient qu'après l'alignement du dernier chromosome: c'est seulement à ce stade que la cohésion entre les chromatides sœurs est rompue. Si la transition M/A avait lieu avant que tous les chromosomes ne soient reliés aux deux pôles du fuseau, cela conduirait inévitablement à une répartition inégale du génome et à la naissance de cellules aneuploïdes, avec des conséquences qui peuvent être catastrophiques pour l'organisme. Cette étape cruciale est sous la surveillance d'un mécanisme appelé point de contrôle de l'intégrité du fuseau, ou point de contrôle mitotique. 
$(\rightarrow) \mathrm{m} / \mathrm{s}$

2002, $n^{\circ} 12$,

p. 1219

\section{La transition M/A: une histoire de protéolyse}

L'entrée en mitose est provoquée par l'activation du MPF (M-phase promoting factor), une protéine kinase hétérodimérique formée d'une sous-unité catalytique, Cdc2, et d'une sous-unité activatrice, la cycline B [2]. La transition $M / A$ coïncide avec la dégradation protéolytique de la cycline $B$ et l'inactivation de Cdc2. La dégradation de la cycline $B$ nécessite en premier lieu son « étiquetage » par des chaînes d'ubiquitine [3], assuré par l'E3 ubiquitine ligase, nommée APC (ana-

phase-promoting complex), en association avec son activateur Cdc20/Fizzy $[4,5]$. La cycline est reconnue par le complexe APCCdc20/Fizzy ( $A P C^{\text {Fizzy }}$ ) grâce à la présence, dans sa séquence, d'un motif oligopeptitique appelé boîte de destruction ( $D-B o x)$ [3]. La cycline $B$ ainsi ubiquitinylée est reconnue et dégradée par le protéasome 26 S (Figure 1). Jusqu'en 1993, l'idée prévalait que seule la dégradation de la cycline B était nécessaire à la transition M/A et aux événements cytologiques qui en découlent. Holloway et Murray [6] ont montré qu'il n'en est rien: le blocage de la machinerie participant à la dégradation de la cycline $B$, par un peptide contenant le motif $D$-Box, stabilise le complexe Cdc2-cycline B (MPF) et empêche également la séparation des chromatides sœurs et la décondensation des chromosomes. En revanche, le maintien de l'activité MPF par une forme stable de la cycline B prévient seulement la décondensation des chromosomes. Ces résultats suggèrent l'existence d'une protéine possédant un motif $D$-Box, mais différente de la cycline B, devant être spécifiquement dégradée pour permettre la séparation des chromatides sœurs [6]. Par la suite, cette protéine a été idenlaires reflète un état activé. tifiée dans de nombreuses espèces, et appelée sécurine [7]. Son rôle est de complexer et d'inhiber une cystéine protéase appartenant à la famille des caspases, la séparase. À la transition M/A, la sécurine est dégradée et libère la séparase, chargée du clivage de la sousunité $\mathrm{Sccl}$ des complexes cohésines, événement essentiel à la perte de cohésion des chromatides sœurs et à leur ségrégation $[8,9]$ (Figure 2) $(\rightarrow)$.

\section{Le point de contrôle mitotique : à chacun son lot}

La phase de capture des chromosomes par les microtubules est un processus aléatoire. Sa durée est donc variable, et peut même prendre plusieurs heures lorsqu'un des chromosomes est mal orienté par rapport aux pôles du fuseau. Durant cette phase, MPF doit rester actif; or, en l'absence de chromosomes, la durée de vie

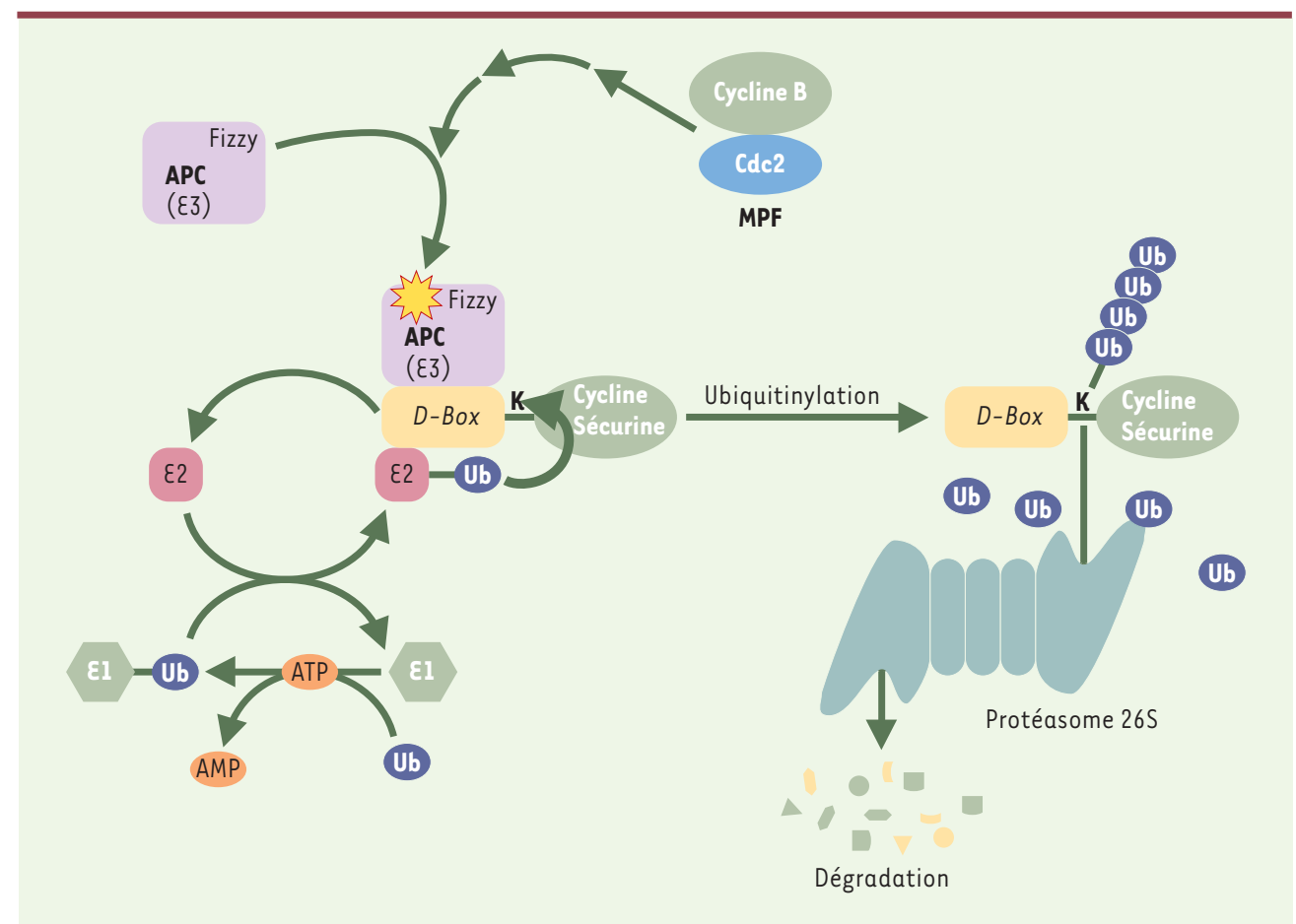

Figure 1. Le complexe APC contrôle l'ubiquitinylation de substrats mitotiques. Les réactions d'ubiquitinylation nécessitent trois types d'enzymes appelées $\varepsilon 1$, $\varepsilon 2$ et $\varepsilon 3$. $\varepsilon$ n principe, $\varepsilon 3$ apporte la spécificité pour la protéine cible de l'ubiquitinylation. Le complexe APC (anaphase-promoting complex) est un édifice moléculaire composé d'au moins 11 sous-unités. II possède une activité $\varepsilon 3$ ubiquitine ligase, et collabore avec l'enzyme d'activation de I'ubiquitine ( $(1)$ et l'enzyme de conjugaison ( $(2)$ pour catalyser le transfert de molécules d'ubiquitine (Ub) sur des résidus lysine de molécules substrats. La réitération de la réaction entraîne l'allongement de la chaîne d'ubiquitine. La protéine Fizzy se lie au complexe APC et l'active. Lorsque la cellule entre en mitose, la kinase mitotique Cdc2-cycline B (ou MPF pour M-phase promoting factor) phosphoryle l'APC, ce qui augmente l'affinité de Fizzy pour l'APC, permettant ainsi la formation de nombreux complexes APC Fizzy actifs, nécessaires à l'ubiquitinylation de la sécurine et de la cycline B au cours de la mitose. Ces protéines ainsi «étiquetées » sont reconnues et rapidement dégradées en petits peptides par le protéasome 26S. Le symbole jaune appliqué sur les entités molécu- 
de MPF est limitée par le fait qu'il est lui-même à l'origine de l'activation de la voie de dégradation de la cycline $B$, sa sous-unité activatrice [10]. Il existe donc nécessairement un mécanisme de couplage entre la phase de capture des chromosomes et la phase d'inactivation de MPF: il s'agit du point de contrôle mitotique. D'une façon générale, les points de contrôle sont des mécanismes élaborés par la cellule pour asservir le déclenchement d'un événement $B$ à la terminaison d'un événement $A$, les deux événements étant mécanistiquement indépendants. En l'occurrence, le point de contrôle mitotique prolonge la durée de vie du MPF tant que tous les chromosomes ne sont pas alignés, assurant le partage des chromosomes en deux lots identiques entre les cellules filles.

Expérimentalement, ce point de contrôle est révélé par l'arrêt en prométaphase de cellules exposées à des poisons des microtubules qui provoquent leur dépolymérisation, comme le nocodazole ou le bénomyl (les chromosomes ne peuvent s'aligner en raison de l'absence de fuseau). Des expériences de criblage génétique menées chez la levure Saccharomyces cerevisiae ont pour la première fois permis d'identifier différents gènes impliqués dans la voie de signalisation de ce point de contrôle.
Des mutants de délétion de chacun des gènes madl, mad2, mad3 (mitotic arrest deficient) [11] et bubl et bub3 (budding uninhibited by benomyl) [12] ne s'arrêtent pas en mitose malgré la présence d'agents dépolymérisateurs des microtubules. La protéine kinase Mpsl, nécessaire pour la duplication du spindle pole body, équivalent fonctionnel du centrosome chez la levure, a par la suite été identifiée comme un composant essentiel du point de contrôle mitotique [13]. Sa surexpression provoque un arrêt en métaphase. Cet arrêt nécessite la fonction des produits de chacun des gènes mad et bub [14]. Mpsl excepté, ces gènes ne sont pas essentiels chez la levure, leur délétion n'empêchant pas la multiplication des cellules.

L'identification d'homologues fonctionnels de ces gènes suggère que le mécanisme de base de ce point de contrôle est conservé chez les eucaryotes supérieurs, bien que d'autres protéines essentielles à son fonctionnement aient été recensées, telles que la protéine kinase BubRl (hybride entre la protéine kinase Bubl et Mad3) [15], la kinésine CENP- $\varepsilon$ (centromeric protein $\varepsilon$ ) [16], les protéines Zeste white 10 (Zw10) et Rough Deal (Rod) $[17,18]$, ainsi qu'une forme de MAP-kinase (microtubule associated protein-kinase) [19].

Le lien a pu être fait entre le point de contrôle mitotique et la machine-

rie de dégradation de la cycline B grâce à la mise en

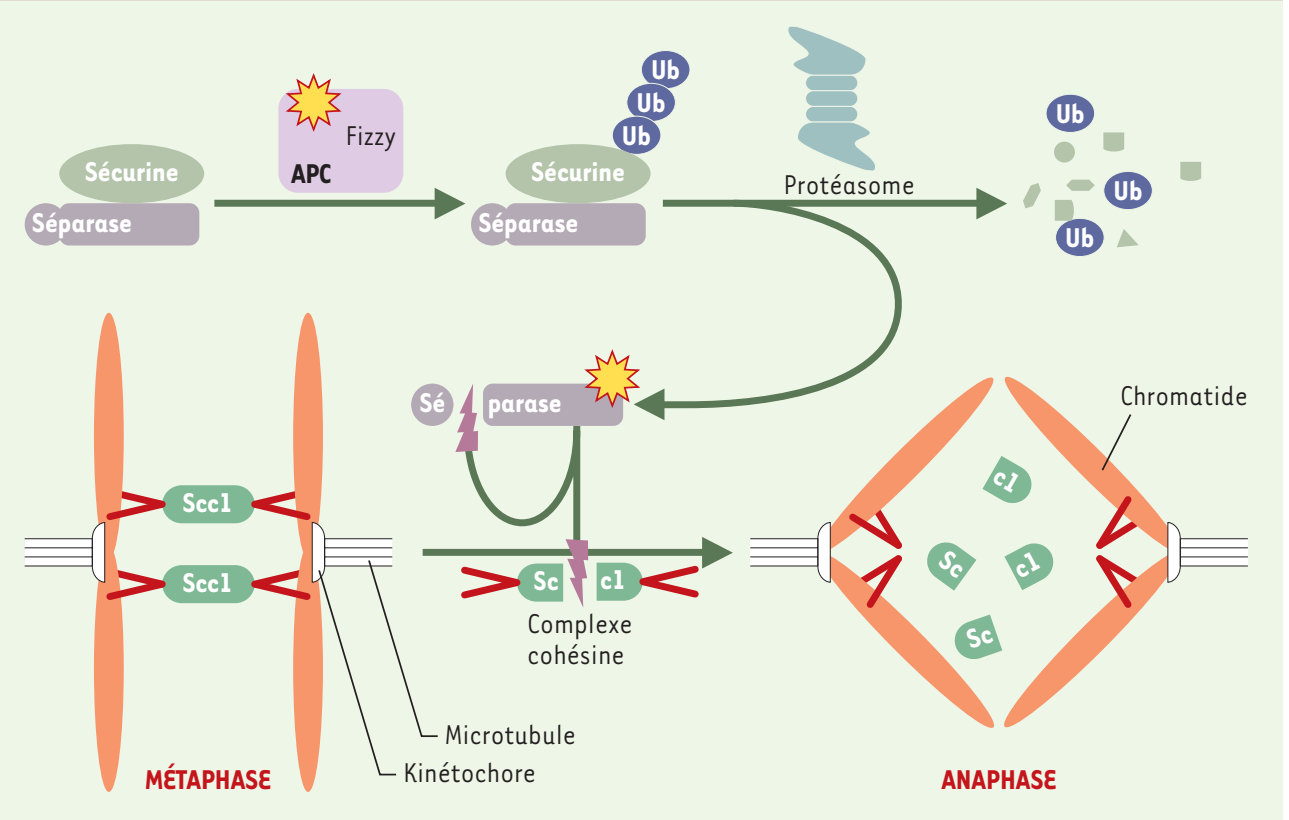

Figure 2. Le complexe APC Fizzy règle la transition métaphase/anaphase. La séparation des chromatides sœurs au cours de la mitose est étroitement réglée pour assurer aux deux cellules filles un lot équivalent de chromosomes. Les deux chromatides sœurs sont maintenues associées par des complexes protéiques appelés cohésines. La sécurine est associée à une protéase de la famille des caspases, la séparase, et la maintient inactive. À la transition métaphase/anaphase, le complexe APC Cizzy actif ubiquitinyle (Ub) la sécurine, qui est alors dégradée par le protéasome 26S. La séparase est alors libérée, s'active et clive la sous-unité Sccl du complexe cohésine, autorisant la séparation des chromatides sœurs. Le symbole jaune appliqué sur les entités moléculaires reflète un état activé. évidence d'une interaction physique entre Mad2 et APC dans les cellules HeLa bloquées en prométaphase par le nocodazole, et grâce à la démonstration selon laquelle Mad2 purifié est capable de bloquer la dégradation de la cycline $B$ dans les extraits mitotiques d'œufs de xénope. Enfin, après la démonstration selon laquelle $\mathrm{Cdc} 20$ / Fizzy est le lien physique entre Mad2 et APC [21], et après l'obtention de mutants thermosensibles de S. cerevisiae et de S. pombe incapables d'interagir avec Mad2 et de s'arrêter en mitose en présence de nocodazole [22, 23], Cdc20/Fizzy est apparu comme la cible du système de contrôle sur le système effecteur. 


\section{Le kinétochore: I'œil du juste}

Le point de contrôle mitotique peut détecter des défauts affectant le fuseau de multiples façons, se résumant toutes à une modification de l'état des kinétochores. Chez S. cerevisiae, une perturbation de l'assemblage des kinétochores ou de leur attachement aux microtubules, résultant de mutations dans la séquence centromérique ou dans la protéine $\mathrm{Ctfl} 3$ qui se lie à cette séquence, provoque un arrêt mitotique. Cet arrêt ne se produit que si les gènes mad et bub sont fonctionnels [24]. D'un autre côté, les cellules dépourvues de la protéine $\mathrm{Ndc10}$, sans laquelle les kinétochores ne peuvent se former, ne s'arrêtent pas en mitose quant elles sont traitées par le nocodazole [25]. Dans les cellules Ptkl, la destruction par microchirurgie au laser du kinétochore libre du dernier chromosome retardataire rend impossible son alignement; pourtant, la cellule déclenche l'anaphase, comme si elle se «croyait » en métaphase [26]. L'ensemble de ces données suggère que les kinétochores libres, ou présentant un défaut de liaison aux microtubules, émettent un signal qui empêche la transition M/A (Figure 3).

La localisation subcellulaire des protéines du point de contrôle mitotique est en adéquation avec cette hypothèse [27]. Les protéines Mad et Bub se localisent préférentiellement sur les kinétochores libres, ou encore sur les kinétochores diri- geant le mouvement des chromosomes vers la plaque équatoriale pendant la phase d'alignement. Au contraire, elles sont très peu présentes, voire absentes en ce qui concerne les protéines Mad, sur les kinétochores des chromosomes alignés [28]. La même évolution spatio-temporelle de localisation avait déjà été

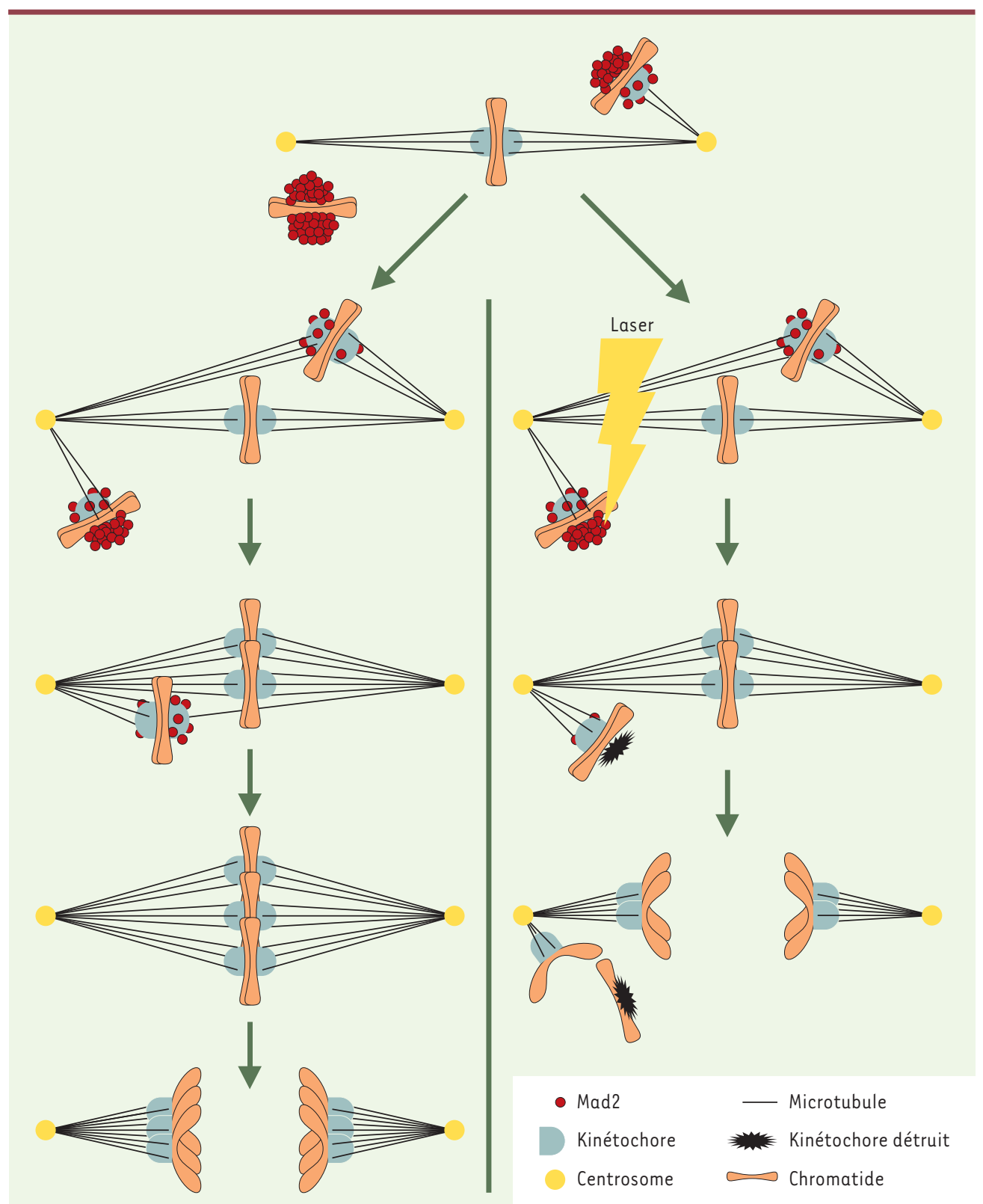

Figure 3. Les kinétochores libres inhibent la transition M/A. Une cellule en mitose ne commence l'anaphase que lorsque le dernier chromosome s'est positionné sur la plaque équatoriale du fuseau (à gauche). Les protéines du point de contrôle se localisent sur les kinétochores, préférentiellement libres, des chromosomes non attachés ou mono-orientés. Lorsqu'un chromosome acquiert un attachement bipolaire, l'abondance de ces protéines diminue progressivement sur le kinétochore qui dirige le déplacement du chromosome vers la plaque équatoriale. En particulier, Mad2 est indétectable sur les kinétochores des chromosomes déjà alignés. La destruction au micro-laser du dernier kinétochore libre déclenche l'anaphase (voie droite). 
décrite pour l'épitope 3F3/2, un phosphoépitope commun à beaucoup de protéines phosphorylées en mitose. Dans les cellules Ptkl, l'injection d'anticorps anti-Mad2 provoque une entrée prématurée en anaphase, montrant que le point de contrôle mitotique fonctionne de façon systématique lors de chaque mitose [29]. Au contraire, l'injection d'anticorps $3 F 3 / 2$ protège son épitope de la déphosphorylation et retarde l'anaphase, suggérant qu'un événement de déphosphorylation est impliqué dans la transition M/A [30].

\section{L'attachement, c'est bien, avec de la tension, c'est mieux}

Chez la mante religieuse, les spermatocytes contiennent trois chromosomes sexuels, un $Y$ et deux $X$ génétiquement différents, associés sous forme d'un chromosome trivalent (Figure 4A). Il arrive parfois qu'un des chromosomes $X$ se détache, et ne soit alors relié qu'à un pôle. Dans ces spermatocytes, la maturation méiotique s'ar-

A

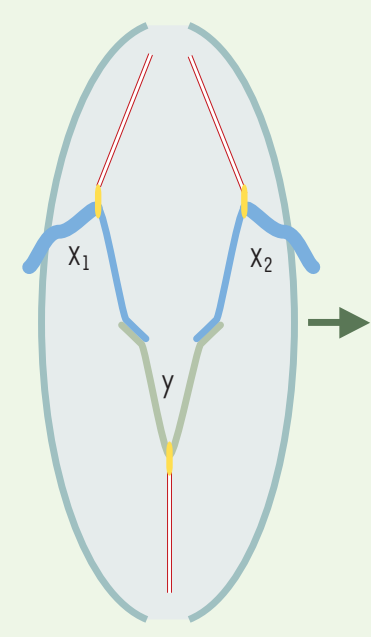

B

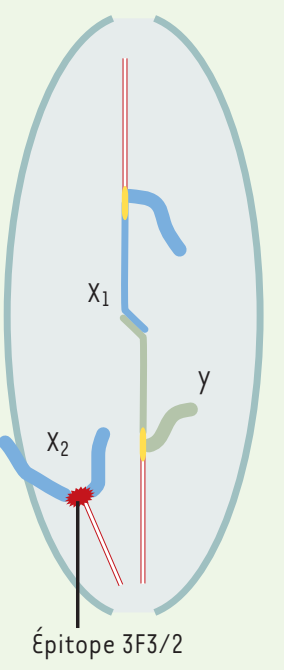

rête en métaphase de première méiose, alors que tous les kinétochores se sont liés à leur complément de microtubules. Cet arrêt est corrélé à la présence de l'épitope $3 \mathrm{~F} 3 / 2$ sur le kinétochore $\mathrm{du} X$ « libre ». X. Li et R.B. Nicklas ont montré qu'il est possible de déclencher l'anaphase dans ces spermatocytes en exerçant une force de traction avec une micro-aiguille sur le chromosome $X$ «libre ». La reprise du cycle est corrélée avec la disparition de l'épitope 3F3/2 [31] (Figure 4). Ces résultats tendent à montrer qu'un point de contrôle sensible à la tension, au niveau des kinétochores, s'exerce dans ces spermatocytes.

Chez S. cerevisiae, certains mutants qui entrent en mitose sans avoir répliqué leur génome ne peuvent en sortir. Dans ces conditions, chaque chromosome ne comporte qu'une seule chromatide, ne possède qu'un kinétochore et ne peut donc acquérir l'attachement bipolaire qui est la source de la tension s'exerçant normalement entre les kinétochores frères. En raison de la morphologie allongée de leur fuseau, ces cellules semblaient bloquées après la transition M/A. Le point de contrôle ne paraissait donc sensible qu'à l'état d'attachement des kinétochores aux microtubules. Or, une étude plus fine de ces mutants a révélé que la sécurine n'était pas dégradée, signifiant que la transition M/A n'avait pas eu lieu. Dans ces mutants, le point de contrôle est donc en activité et sensible à l'absence de tension. Cet arrêt dépend bien de Mad2 et - contrairement à l'arrêt produit par le nocodazole - de la protéine kinase Ipll (appartenant à la famille des protéine kinases Aurora) $[32,33](\rightarrow)$. Ces travaux suggèrent que le point de contrôle mitotique dispose de deux types de «senseurs», l'un sensible à l'état d'attachement des kinétochores, l'autre à la tension.

Cette double sensibilité du point de contrôle semble être un caractère conservé à travers les espèces. Chez la drosophile, on peut dis- 
tinguer les mutants Bubl, dont les cellules entrent en anaphase avant que tous les kinétochores ne soient correctement attachés [34], et les mutants Rod et Zw10, où l'anaphase commence après l'attachement de tous les kinétochores, mais avant l'alignement de tous les chromosomes [18]. Chez les vertébrés, un blocage en métaphase peut également être obtenu par des agents qui altèrent la dynamique du fuseau, sans le détruire. La faible distance séparant alors les kinétochores frères démontre une absence de tension entre eux. Le phosphoépitope 3F3/2 est présent sur tous les kinétochores, contrairement à Mad2 qui ne se localise que sur certains, suggérant que la perte de tension ne suffit pas, en soi, à provoquer cette localisation. L'implication de Mad2 dans le signal inhibiteur provoqué par l'absence de tension est donc actuellement controversée chez les vertébrés $[35,36]$.

\section{La valse des interactions}

D'abondantes interactions entre les protéines du point de contrôle mitotique ont été décrites, laissant supposer l'existence d'importants édifices supramoléculaires dont les fonctions sont encore obscures. On sait avec certitude que Madl interagit avec Mad2, et que l'interaction du complexe Madl-Mad2 avec les kinétochores dépend de Madl. De plus, Mad2 interagit directement avec Cdc20/Fizzy. Chez la levure, cette interaction disparaît dans les mutants de délétion de madl. Bub3 interagit avec Bubl et BubRl, cette interaction étant essentielle à leur localisation sur les kinétochores. Dans les extraits d'œufs de xénope, la localisation du complexe Madl-Mad2 sur les kinétochores dépend du complexe Bub3-Bubl, mais pas de l'activité kinase de Bubl. Elle dépend également de la chromokinésine CENP- $\varepsilon$ et de l'activité de la protéine kinase Mpsl
[37]. CENP- $\varepsilon$ interagit directement avec BubRl. Par ailleurs, les protéines Madl,2 et Bub3, 1, et Rl ont également été trouvées en association avec l'APC [38].

\section{La ballade de Mad2}

Le kinétochore libre semble être le lieu de production d'une forme transitoire de Mad2 capable d'inhiber $A P C^{\text {Fizzy }}$ et la transition M/A (Figure 5). L'étude dynamique du comportement d'une protéine Mad2 fluorescente, surexprimée dans des cellules vivantes, montre que Mad2 s'associe transitoirement aux kinétochores, avec un temps de demi-renouvellement d'environ 20 secondes. De plus, Mad2 transite le long des microtubules, des kinétochores vers les pôles, derniers endroits de l'appareil mitotique que quitte Mad2 avant la transition M/A [39]. Quand le point de contrôle est activé par le nocodazole, Mad2 se localise uniquement sur les kinétochores, ce qui suggère que sa présence sur les pôles dépend exclusivement de son transport sur les microtu-

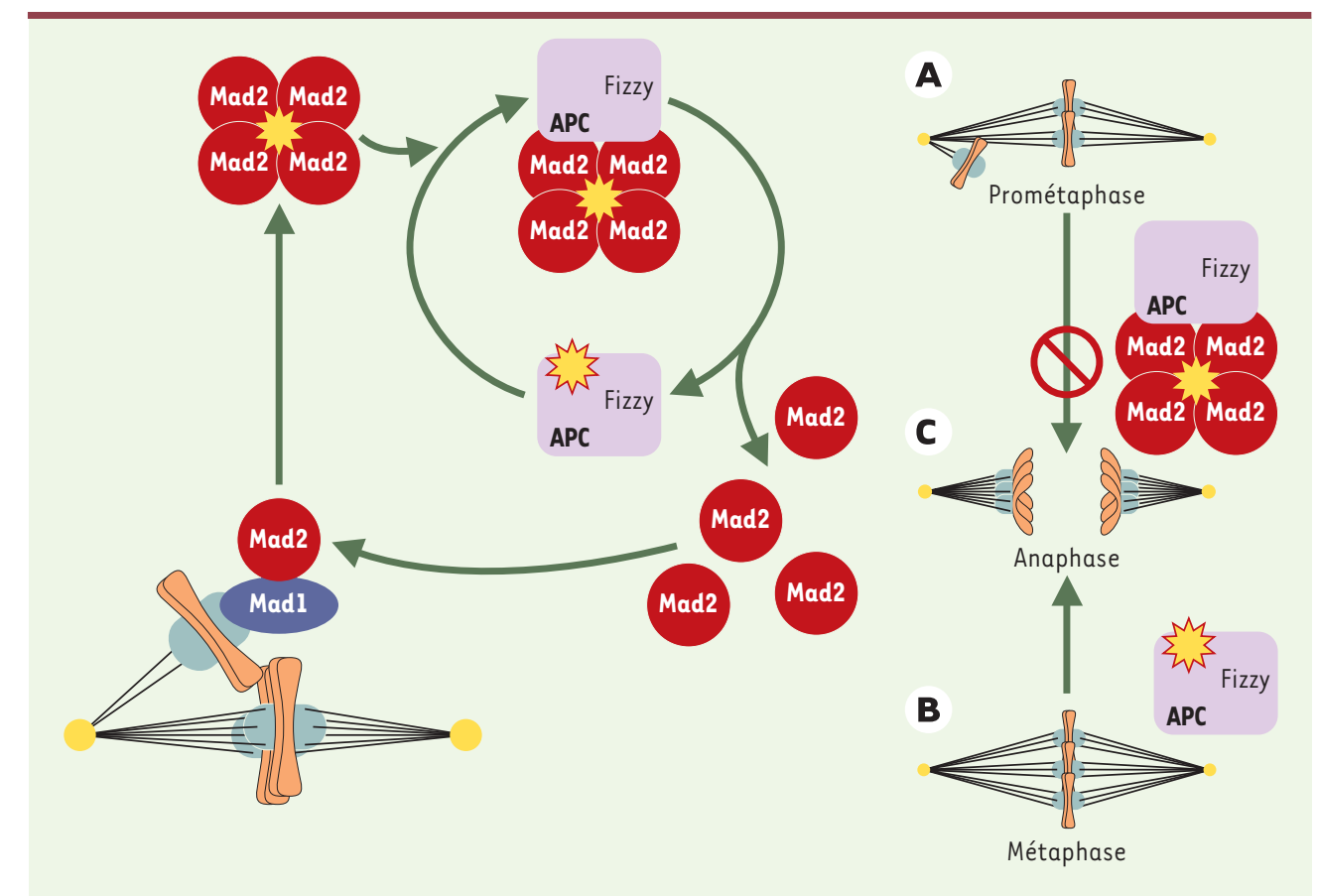

Figure 5. Modèle fonctionnel de base du point de contrôle mitotique. Ce modèle s'appuie sur quatre données majeures: le kinétochore libre émet un signal inhibiteur, Mad2 sous forme tétramérique inhibe l'activité d'ubiquitinylation de l'APC, alors qu'une forme monomérique en est incapable, Mad2 se lie directement à Cdc20/Fizzy, et des mutants de $\mathrm{Cdc} 20$, chez S. cerevisiae, incapables de se lier à Mad2, ont un point de contrôle défectueux. Dans ce modèle, le kinétochore libre est une plate-forme de production d'un signal inhibiteur de la transition métaphase/anaphase. En prométaphase ( $\boldsymbol{A}$, et agrandissement à gauche sur le schéma), les kinétochores libres se lient à Mad2 via Madl, ce qui aboutit à la formation d'une forme transitoirement activée de Mad2 capable de se lier et d'inhiber APC $C^{\text {fizzy }}$, ce qui empêche la transition M/A. Une fois tous les chromosomes alignés sur la plaque équatoriale (B), la forme inhibitrice de Mad2 cesse d'être produite, APC Fizzy n'est plus inhibé et la transition M/A a lieu (C). Le symbole jaune appliqué sur les entités moléculaires reflète un état activé. 
bules. Les protéines Rod et $\mathrm{Zwl} 0$ sont essentielles au fonctionnement du mécanisme de surveillance chez les eucaryotes supérieurs. Elles sont nécessaires au recrutement de la dynéine au niveau des kinétochores. La dynéine est un moteur moléculaire se déplaçant vers l'extrémité « moins » des microtubules et est vraisemblablement impliquée dans le transport de Mad2.

Lorsque deux fuseaux sont présents dans une même cellule, le premier fuseau à atteindre la métaphase entre en anaphase. II provoque alors une anaphase prématurée du second fuseau, même si celui-ci présente encore plusieurs chromosomes mono-orientés, c'est-à-dire attachés à un seul pôle. Ces observations laissent penser que la transmission du signal inhibiteur produit par le kinétochore libre d'un chromosome mono-orienté est liée à sa connexion au fuseau, et qu'un signal positif dominant est à l'origine de la transition M/A [40]. Dans les cellules HeLa, la cycline B disparaît d'abord sur le fuseau et les chromosomes [41]. Chez la drosophile, la destruction de la cycline B commence d'abord aux niveaux des pôles, s'étend ensuite au reste du fuseau [42] et dépend de la connexion centrosomes/fuseau [43]. En effet, chez les mutants Cfo (centrosome fall off), dont les centrosomes se détachent du fuseau après la transition M/A, la destruction de la cycline B continue sur les centrosomes et s'arrête sur le reste du fuseau. D'un côté, Mad2 transite des kinétochores vers les pôles, semblant centraliser l'information émise par tous les chromosomes qui lui sont liés, et maintenir le point de contrôle en activité. D'un autre côté, le centrosome apparaît comme le lieu d'amorçage des dégradations dépendant de l'APC Fizzy , la propagation de ces dégradations au reste de la cellule dépendant de la liaison entre centrosome et fuseau. Un complexe entre BubRl-Bub3Mad2-Cdc20 a récemment été purifié, à partir d'extraits cellulaires, sur la base d'une activité capable d'inhiber I'APC. Ces complexes existent quelle que soit la phase du cycle cellulaire, à des moments où les kinétochores ne sont pas encore matures [44]. À l'inverse, seule une forme mitotique de I'APC semble sensible à leur activité inhibitrice, suggérant que le mécanisme de surveillance contrôle la sensibilité de l'APC à l'inhibiteur, plutôt que l'inhibiteur lui-même.

\section{La socialisation cellulaire et la nécessité du partage}

Curieusement, le point de contrôle mitotique était initialement perçu comme un mécanisme activé occasionnellement en cas de défaillance de l'appareil mitotique, alors que la notion de dernier kinétochore libre inhibiteur de la transition M/A implique un mécanisme fonc- tionnant de façon systématique. Cette vision était largement due aux études menées sur les mutants de délétion de ces gènes chez la levure. Ces mutants se divisent sans que la transition M/A ne semble avoir lieu prématurément, mais leur descendance présente un taux anormalement élevé d'aneuploïdie.

Au contraire, chez les métazoaires, un point de contrôle mitotique défectueux provoque une entrée prématurée en anaphase [29], avec des conséquences d'autant plus graves que la survie cellulaire n'est pas un critère suffisant à la survie de l'organisme. Les pertes de fonction de Bubl chez la drosophile, et celles de Mad2 et Bub3 chez la souris, sont létales très tôt au cours du développement embryonnaire $[36,45]$. Dans un organisme déjà développé, la mort cellulaire en cas d'aneuploïdie est ce qu'il y a de plus souhaitable. En effet, la plupart des tumeurs présentent une instabilité génétique au niveau chromosomique. Il apparaît de plus en plus que des défaillances du point de contrôle mitotique sont à l'origine de cette instabilité, sans doute elle-même impliquée dans le processus de cancérisation [46] $(\rightarrow)$. Les protéines humaines $\mathrm{HsBubl}$ et $\mathrm{HsBubRl}$ ont été trouvées mutées dans des cellules de cancer du côlon [47], HsMadl interagit avec la protéine Tax du virus HTLV-1, impliquée dans certaines leucémies [48]. La quantité de HsMad2 est fortement diminuée dans des lignées cellulaires provenant de cancers du sein [49]. Mais, plus surprenant, les souris transgéniques ne portant qu'une seule copie fonctionnelle du gène HsMAD2 développent un taux anormal de tumeurs [50]. Une compréhension globale des mécanismes moléculaires impliqués dans le point de contrôle mitotique, mais aussi des autres mécanismes de surveillance impliqués dans le contrôle du cycle cellulaire, devrait multiplier les possibilités d'action sur la prolifération cellulaire et augmenter le choix des stratégies thérapeutiques. $\diamond$

\section{REMERCIEMENTS}

Les travaux de notre équipe sont soutenus par la Ligue nationale contre le cancer: équipe labellisée. $(\rightarrow) \mathrm{m} / \mathbf{s}$

2002, $n^{\circ} 12$, p. 1227 


\section{SUMMARY}

\section{Mitosis under control}

The mitotic checkpoint is essential to ensure accurate chromosome segregation by allowing a mitotic delay in response to a spindle defect. This checkpoint postpones the onset of anaphase until all the chromosomes are attached and correctly aligned onto the mitotic spindle. The checkpoint functions by preventing an ubiquitin ligase called the anaphase-promoting complex (APC) from ubiquitinylating proteins whose degradation is required for anaphase onset. Loss of this checkpoint results in chromosome missegregation in higher eukaryotes and may contribute to the genomic instability observed in most of the tumour cells. $\diamond$

\section{RÉFÉRENCES}

1. Karsenti $\varepsilon$, Vernos I. The mitotic spindle: a selfmade machine. Science 2001; 294: 543-7.

2. Labbé JC, Capony JP, Caput D, et al. MPF from starfish oocytes at first meiotic metaphase is a heterodimer containing one molecule of cdc2 and one molecule of cyclin B. $\varepsilon M B O$ J 1989; 8: 3053-8.

3. Glotzer M, Murray AW, Kirschner MW. Cyclin is degraded by the ubiquitin pathway. Nature 1991; 349: 132-8.

4. Peters JM. Subunits and substrates of the anaphase-promoting complex. Exp Cell Res 1999; 248: 339-49.

5. Lorca T, Castro A, Martinez $A M$, et al. Fizzy is required for activation of the $\mathrm{APC} /$ cyclosome in Xenopus egg extracts. EMBO J 1998; 17: 3565-75.

6. Holloway SL, Glotzer M, King RW, Murray AW. Anaphase is initiated by proteolysis rather than by the inactivation of maturation-promoting factor. Cell 1993; 73 : 1393-402.

7. Zou H, McGarry TJ, Bernal T, Kirschner MW. Identification of a vertebrate sisterchromatid separation inhibitor involved in transformation and tumorigenesis. Science 1999; 285: 418-22.

8. Hirano T. Chromosome cohesion, condensation, and separation. Annu Rev Biochem 2000; 69: 115-44.

9. Uhlmann F, Lottspeich F, Nasmyth K. Sisterchromatid separation at anaphase onset is promoted by cleavage of the cohesin subunit $\mathrm{Sccl}$. Nature 1999; 400: 37-42.

10. Felix MA, Labbé JC, Dorée $M$, Hunt $T$, Karsenti $\varepsilon$. Triggering of cyclin degradation in interphase extracts of amphibian eggs by cdc2 kinase. Nature 1990; 346: 379-82.

11. Li R, Murray AW. Feedback control of mitosis in budding yeast. Cell 1991; 66: 519-31.

12. Hoyt MA, Totis L, Roberts BT. S. cerevisiae genes required for cell cycle arrest in response to loss of microtubule function. Cell 1991; 66: 507-17.

13. Weiss $\varepsilon$, Winey M. The Saccharomyces cerevisiae spindle pole body duplication gene MPS1 is part of a mitotic checkpoint. J Cell Biol 1996; 132: 111-23.

14. Hardwick KG, Weiss E, Luca FC, Winey M, Murray AW. Activation of the budding yeast spindle assembly checkpoint without mitotic spindle disruption. Science
1996; 273: 953-6.

15. Taylor SS, Ha E, McKeon F. The human homologue of Bub3 is required for kinetochore localization of Bubl and a Mad3/Bublrelated protein kinase. J Cell Biol 1998; 142: 1-11.

16. Abrieu A, Kahana JA, Wood KW, Cleveland DW. CENP- $\varepsilon$ as an essential component of the mitotic checkpoint in vitro. Cell 2000; 102 : 817-26.

17. Chan GK, Jablonski SA, Starr DA, Goldberg ML, Yen TJ. Human Zw10 and ROD are mitotic checkpoint proteins that bind to kinetochores. Nat Cell Biol $2000 ; 2: 944-7$.

18. Basto R, Gomes R, Karess RE. Rough deal and $Z w 10$ are required for the metaphase checkpoint in Drosophila. Nat Cell Biol 2000; 2 : 939-43.

19. Minshull J, Sun H, Tonks NK, Murray AW. A MAP kinasedependent spindle assembly checkpoint in Xenopus egg extracts. Cell 1994; 79: 475-86.

20. Li Y, Gorbea C, Mahaffey D, Rechsteiner M, Benezra R. MAD2 associates with the cyclosome/anaphasepromoting complex and inhibits its activity. Proc Natl Acad Sci USA 1997; 94: 12431-6.

21. Kallio M, Weinstein J, Daum JR, Burke DJ, Gorbsky GJ. Mammalian p55CDC mediates association of the spindle checkpoint protein Mad2 with the cyclosome/anaphasepromoting complex, and is involved in regulating anaphase onset and late mitotic events. J Cell Biol 1998; 141: 1393-406.

22. Hwang LH, Lau LF, Smith $\mathrm{DL}$, et al. Budding yeast Cdc20: a target of the spindle checkpoint. Science 1998; 279: 1041-4.

23. Kim SH, Lin DP, Matsumoto $S$, Kitazono A, Matsumoto T. Fission yeast SIpl: an effector of the Mad2dependent spindle checkpoint. Science 1998; 279: 1045-7.
24. Wang $y$, Burke DJ. Checkpoint genes required to delay cell division in response to nocodazole respond to impaired kinetochore function in the yeast Saccharomyces cerevisiae. Mol Cell Biol 1995; 15: 6838-44.

25. Tavormina PA, Burke DJ. Cell cycle arrest in cdc20 mutants of Saccharomyces cerevisiae is independent of Ndc 10p and kinetochore function but requires a subset of spindle checkpoint genes. Genetics 1998; 148: 1701-13.

26. Rieder CL, Cole RW, Khodjakov A, Sluder G. The checkpoint delaying anaphase in response to chromosome

monoorientation is mediated by an inhibitory signal produced by unattached kinetochores. J Cell Biol 1995; 130: 941-8.

27. Dobie KW, Hari KL, Maggert KA, Karpen GH. Centromere proteins and chromosome inheritance: a complex affair. Curr Opin Genet Dev 1999; 9: 206-17.

28. Hoffman DB, Pearson CG, Yen TJ, Howell BJ, Salmon $\varepsilon D$. Microtubule-dependent changes in assembly of microtubule motor proteins and mitotic spindle checkpoint proteins at PtKl kinetochores. Mol Biol Cell 2001; 12: 1995-2009.

29. Gorbsky GJ, Chen RH, Murray AW. Microinjection of antibody to Mad2 protein into mammalian cells in mitosis induces premature anaphase. J Cell Biol 1998; 141: 1193-205.

30. Campbell MS, Gorbsky GJ. Microinjection of mitotic cells with the $3 \mathrm{~F} 3 / 2$ antiphosphoepitope antibody delays the onset of anaphase. J Cell Biol 1995. 129: 1195-204.

31. Li X, Nicklas RB. Tensionsensitive kinetochore phosphorylation and the chromosome distribution checkpoint in praying mantid spermatocytes. J Cell Sci 1997; 110: 537-45. 
32. Stern BM, Murray AW. Lack of tension at kinetochores activates the spindle checkpoint in budding yeast. Curr Biol 2001; 11: 1462-7.

33. Biggins S, Murray AW. The budding yeast protein kinase Ipl1/Aurora allows the absence of tension to activate the spindle checkpoint. Genes Dev 2001; 15: 3118-29.

34. Basu J, Bousbaa H, Logarinho $\varepsilon$, et al. Mutations in the essential spindle checkpoint gene bubl cause chromosome missegregation and fail to block apoptosis in Drosophila. J Cell Biol 1999; 146: 13-28.

35. Waters JC, Chen RH, Murray AW, Salmon ED. Localization of Mad2 to kinetochores depends on microtubule attachment, not tension. J Cell Biol 1998; 141: 1181-91.

36. Skoufias DA, Andreassen PR, Lacroix FB, Wilson L, Margolis RL. Mammalian mad2 and bubl/bubRl recognize distinct spindle-attachment and kinetochore-tension checkpoints. Proc Natl Acad Sci USA 2001; 98: 4492-7.

37. Abrieu A, Magnaghi-Jaulin L, Kahana JA, et al. Mpsl is a kinetochore-associated kinase essential for the vertebrate mitotic checkpoint. Cell 2001; 106 : 83-93.

38. Wassmann K, Benezra R. Mitotic checkpoints: from yeast to cancer. Curr Opin Genet Dev 2001; 11: 83-90.

39. Howell BJ, Hoffman DB, Fang G, Murray AW, Salmon ED. Visualization of Mad2 dynamics at kinetochores, along spindle fibers, and at spindle poles in living cells. J Cell Biol 2000; 150: 1233-50.

40. Rieder CL, Khodjakov A, Paliulis LV, Fortier TM, Cole RW, Sluder G. Mitosis in vertebrate somatic cells with two spindles: implications for the metaphase/anaphase transition checkpoint and cleavage. Proc Natl Acad Sci USA 1997; 94: 5107-12.
41. Clute P, Pines J. Temporal and spatial control of cyclin $\mathrm{Bl}$ destruction in metaphase. Nat Cell Biol 1999; 1: 82-7.

42. Huang J, Raff JW. The disappearance of cyclin B at the end of mitosis is regulated spatially in Drosophila cells. EMBO J 1999; 18: 2184-95.

43. Wakefield JG, Huang JY, Raff JW. Centrosomes have a role in regulating the destruction of cyclin B in early Drosophila embryos. Curr Biol 2000; 10: 367-70.

44. Sudakin V, Chan GK, Yen TJ. Checkpoint inhibition of the $\mathrm{APC} / \mathrm{C}$ in HeLa cells is mediated by a complex of BUBR1, BUB3, CDC20, and MAD2. J Cell Biol 2001; 154: 925-36.

45. Dobles M, Liberal V, Scott ML, Benezra R, Sorger PK. Chromosome missegregation and apoptosis in mice lacking the mitotic checkpoint protein Mad2. Cell 2000; 101: 635-45.
46. Lengauer C, Kinzler KW, Vogelstein B. Genetic instabilities in human cancers. Nature 1998; 396: 643-9.

47. Cahill DP, Lengauer C, $Y_{u}$ J, et al. Mutations of mitotic checkpoint genes in human cancers. Nature 1998; 392 : 300-3.

48. Jin DY, Spencer F, Jeang KT. Human T cell leukemia virus type 1 oncoprotein Tax targets the human mitotic checkpoint protein MADI. Cell 1998; 93: 81-91.

49. Li Y, Benezra R. Identification of a human mitotic checkpoint gene: hsMAD2. Science 1996; 274 : 246-8.

50. Michel LS, Liberal V, Chatterjee A, et al. MAD2 haplo-insufficiency causes premature anaphase and chromosome instability in mammalian cells. Nature 2001; 409: 355-9.

\section{TIRÉS À PART}

A. Castro 